\title{
SYLVESTER EQUATIONS FOR LAGUERRE-HAHN ORTHOGONAL POLYNOMIALS ON THE REAL LINE
}

\author{
A. BRANQUINHO, A. PAIVA AND M.N. REBOCHO
}

\begin{abstract}
Matrix Sylvester differential equations are introduced in the study of Laguerre-Hahn orthogonal polynomials. Matrix Sylvester differential systems are shown to yield representations for the Laguerre-Hahn orthogonal polynomials. Lax pairs are given, formed from the differential system and the recurrence relation, that yield discrete non-linear equations for the three term recurrence relation coefficients of the Laguerre-Hahn orthogonal polynomials.
\end{abstract}

KEYWORDS: Orthogonal polynomials on the real line, Stieltjes functions, Riccati differential equations, matrix Sylvester differential equations.

AMS Subject Classification (2000): 33C47, 42C05.

\section{Introduction}

Laguerre-Hahn orthogonal polynomials arise in several subjects of mathematics, such as probability theory [9], differential equations [8, 14, 17], measure perturbation theory [6], constructive theory of approximation [11].

From an analytical point of view, Laguerre-Hahn orthogonal polynomials can be regarded as a generalization of semi-classical orthogonal polynomials, since they are related to Stieltjes functions that are solutions of Riccati differential equations

$$
A S^{\prime}=B S^{2}+C S+D,
$$

where $A-D$ are polynomials (note that in the semi-classical case there holds $B \equiv 0)[1,7,15]$.

In this paper we present a study of the Laguerre-Hahn families of orthogonal polynomials in terms of matrix Sylvester differential equations. Such a connection is established by means of the equivalence between (1) and the matrix Sylvester differential equations

$$
A Y_{n}^{\prime}=\mathcal{B}_{n} Y_{n}-Y_{n} \mathcal{C}, \quad n \geq 0,
$$

Received July 12, 2012.

This work was supported by Centre for Mathematics of the University of Coimbra, and Fundação para a Ciência e Tecnologia (Portugal), through European program COMPETE/FEDER. M.N. Rebocho was also supported by grant ref. SFRH/BPD/45321/2008, from Fundação para a Ciência e Tecnologia (Portugal). 
where $Y_{n}=\left[\begin{array}{cc}P_{n+1} & P_{n}^{(1)} \\ P_{n} & P_{n-1}^{(1)}\end{array}\right]$, with $\left\{P_{n}\right\}$ the sequence of monic orthogonal polynomials related to $S$ and $\left\{P_{n}^{(1)}\right\}$ the corresponding sequence of associated polynomials (also known as the numerator polynomials), and $\mathcal{B}_{n}, \mathcal{C}$ matrices with polynomial entries. The theory of matrix Sylvester differential equations [16] provides a representation for the solutions of (2) in terms of the solutions of two first order linear systems, which we will relate to the semi-classical orthogonal polynomials.

Our first main result is contained in Theorem 1, where we show the equivalence between (1), (2),

$$
A Q_{n}^{\prime}=\left(\mathcal{B}_{n}+(B S+C / 2) I\right) Q_{n}, \quad Q_{n}=\left[\begin{array}{c}
q_{n+1} \\
q_{n}
\end{array}\right], \quad n \geq 0,
$$

and

$$
A \mathcal{A}_{n}^{\prime}=\mathcal{B}_{n} \mathcal{A}_{n}-\mathcal{A}_{n} \mathcal{B}_{n-1}, \quad \mathcal{A}_{n}=\left[\begin{array}{cc}
x-\beta_{n} & -\gamma_{n} \\
1 & 0
\end{array}\right], \quad n \geq 1
$$

with $q_{n}$ the functions of the second kind, $I$ the identity matrix, and where $\beta_{n}, \gamma_{n}$ are the recurrence relation coefficients of $\left\{P_{n}\right\}$.

As a consequence of the previous equivalence, we obtain that a necessary and sufficient condition for a sequence of monic polynomials $\left\{P_{n}\right\}$, orthogonal with respect to a weight $w$, to be semi-classical is that the following differential system holds (cf. Theorem 2):

$$
A \widetilde{Y}_{n}^{\prime}=\left(\mathcal{B}_{n}-C / 2 I\right) \tilde{Y}_{n}, \quad \widetilde{Y}_{n}=\left[\begin{array}{cc}
P_{n+1} & q_{n+1} / w \\
P_{n} & q_{n} / w
\end{array}\right], \quad n \geq 1,
$$

where $C$ is a polynomial (similar differential systems had been studied, for example, in [13, Section 3]). Such a characterization for semi-classical orthogonal polynomials allows us to deduce a representation for the solutions of $(2)$ as $Y_{n}=\widetilde{\mathcal{P}}_{n} \mathcal{L}^{-1}$, where $\mathcal{L}$ satisfies $A \mathcal{L}^{\prime}=\mathcal{C} \mathcal{L}$ and $\widetilde{\mathcal{P}}_{n}$ is defined in terms of a semi-classical family, say $\left\{\tilde{P}_{n}\right\}$ (cf. Lemma 3 and Theorem 4 ). Furthermore, it is shown that the Stieltjes functions related to $\left\{P_{n}\right\}$ and $\left\{\tilde{P}_{n}\right\}$ are a fractional linear transformation of each other (cf. Lemma 3).

The above referred results will be applied to the study of the LaguerreHahn family of class zero, i.e., $\max \{\operatorname{deg}(A), \operatorname{deg}(B)\} \leq 2$ and $\operatorname{deg}(C)=1$ in (1): in the Theorem 5 we show the solutions of the equation (4) in closed form expressions for the recurrence relation coefficients; in the Theorem 6 
we show a representation of a given sequence of Laguerre-Hahn orthogonal polynomials.

Let us emphasize that the equations (4), enclosing nonlinear difference equations for the recurrence coefficients $\beta_{n}$ and $\gamma_{n}$, are given by the Lax pair

$$
Y_{n}=\mathcal{A}_{n} Y_{n-1}, \quad A Y_{n}^{\prime}=\mathcal{B}_{n} Y_{n}-Y_{n} \mathcal{C} .
$$

The equations (4) can be viewed as comparable to the so-called LaguerreFreud's equations that hold for semi-classical families [12]. There are studies on the recurrence coefficients of (semi-classical) orthogonal polynomials showing its relevance in the theory of integrable systems and Painlevé equations, and we refer the reader to [13] and the references therein (see also [4]).

This paper is organized as follows. In Section 2 we give the definitions and state the basic results which will be used in the forthcoming sections. In Section 3 we establish the equivalence between $(1),(2),(3),(4)$, and we deduce the characterization for semi-classical orthogonal polynomials in terms of first order differential systems. In Section 4 we study the representation of Laguerre-Hahn orthogonal polynomials. The Section 5 is devoted to the study of Laguerre-Hahn orthogonal polynomials of class zero.

\section{Preliminary Results}

Let $\mathbb{P}=\operatorname{span}\left\{z^{k}: k \in \mathbb{N}_{0}\right\}$ be the space of polynomials with complex coefficients, and let $\mathbb{P}^{\prime}$ be its algebraic dual space. We will denote by $\langle u, f\rangle$ the action of $u \in \mathbb{P}^{\prime}$ on $f \in \mathbb{P}$.

Given the moments of $u, u_{n}=\left\langle u, x^{n}\right\rangle, n \geq 0$, where we take $u_{0}=1$, the principal minors of the corresponding Hankel matrix are defined by $H_{n}=$ $\operatorname{det}\left(\left(u_{i+j}\right)_{i, j=0}^{n}\right), n \geq 0$, where, by convention, $H_{-1}=1$. $u$ is said to be quasidefinite (respectively, positive-definite) if $H_{n} \neq 0$ (respectively, $H_{n}>0$ ), for all $n \geq 0$.

Definition 1. (see [18]) Let $u \in \mathbb{P}^{\prime}$ and let $\left\{P_{n}\right\}_{n \geq 0}$ be a sequence of polynomials such that $\operatorname{deg}\left(P_{n}\right)=n, n \geq 0 .\left\{P_{n}\right\}$ is said to be a sequence of orthogonal polynomials with respect to $u$ if

$$
\left\langle u, P_{n} P_{m}\right\rangle=h_{n} \delta_{n, m}, h_{n}=\left\langle u, P_{n}^{2}\right\rangle \neq 0, \quad n, m \geq 0 .
$$

Throughout the paper we shall take each $P_{n}$ monic, that is, $P_{n}(z)=z^{n}+$ lower degree terms, and we will denote $\left\{P_{n}\right\}$ by SMOP.

The equivalence between the quasi-definiteness of $u \in \mathbb{P}^{\prime}$ and the existence of a SMOP with respect to $u$ is well-known in the literature of orthogonal 
polynomials (see $[5,18]$ ). Furthermore, if $u$ is positive-definite, then it has an integral representation in terms of a positive Borel measure, $\mu$, supported on an infinite set of points of the real line, $I$, such that

$$
\left\langle u, x^{n}\right\rangle=\int_{I} x^{n} d \mu(x), \quad n \geq 0,
$$

and the orthogonality condition (5) becomes

$$
\int_{I} P_{n}(x) P_{m}(x) d \mu(x)=h_{n} \delta_{n, m}, \quad h_{n}>0, \quad n, m \geq 0 .
$$

Further, if $\mu$ is defined in terms of a weight $w, d \mu(x)=w(x) d x$, then we will also say that $\left\{P_{n}\right\}$ is orthogonal with respect to $w$.

Monic orthogonal polynomials satisfy a three term recurrence relation [18]

$$
P_{n+1}(x)=\left(x-\beta_{n}\right) P_{n}(x)-\gamma_{n} P_{n-1}(x), \quad n=1,2, \ldots
$$

with $P_{0}(x)=1, P_{1}(x)=x-\beta_{0}$ and $\gamma_{n} \neq 0, n \geq 1, \gamma_{0}=1$.

Definition 2. Let $\left\{P_{n}\right\}$ be the SMOP with respect to a linear functional $u$. The sequence of associated polynomials is defined by

$$
P_{n}^{(1)}(x)=\left\langle u_{t}, \frac{P_{n+1}(x)-P_{n+1}(t)}{x-t}\right\rangle, \quad n \geq 0,
$$

where $u_{t}$ denotes the action of $u$ on the variable $t$.

Definition 3. The Stieltjes function of $u \in \mathbb{P}^{\prime}$ is defined in terms of the moments, $\left(u_{n}\right)$, of $u$ by $S(x)=-\sum_{n=0}^{+\infty} \frac{u_{n}}{x^{n+1}}$.

$S$ has an expansion in terms of a continued fraction, given by

$$
S(x)=\frac{1}{x-\beta_{0}-\frac{\gamma_{1}}{x-\beta_{1}-\frac{\gamma_{2}}{\ddots}}}
$$

where the $\gamma$ 's and the $\beta$ 's are the three term recurrence relation coefficients of the corresponding SMOP. Note that if $u$ is positive-definite, defined by (6), then $S$ is given by $S(x)=\int_{I} \frac{d \mu(t)}{x-t}, \quad x \in \mathbb{C} \backslash I$.

The sequence of functions of the second kind corresponding to $\left\{P_{n}\right\}$ is defined as follows:

$$
q_{n+1}=P_{n+1} S-P_{n}^{(1)}, \quad n \geq 0, q_{0}=S .
$$


Definition 4. (see [15]) A Stieltjes function, $S$, is said to be Laguerre-Hahn if there exist polynomials $A, B, C, D$, with $A \neq 0$, such that it satisfies a Riccati differential equation

$$
A S^{\prime}=B S^{2}+C S+D .
$$

The corresponding sequence of orthogonal polynomials is called LaguerreHahn. If $B=0$, then $S$ is said to be Laguerre-Hahn affine or semi-classical.

If $S$ is related to a positive-definite linear functional defined in terms of a weight, $w$, then the semi-classical character of $S$ means $w^{\prime} / w=C / A$, with $A, C$ polynomials, and such a differential equation is equivalent to $A S^{\prime}=$ $C S+D$, where $D$ is a polynomial given in terms of $A, C$ (see [15]).

Note that (9) is equivalent to the distributional equation $[7,15]$

$$
\mathcal{D}(A u)=\psi u+B\left(x^{-1} u^{2}\right), \quad \psi=A^{\prime}+C .
$$

In the sequel we will use the following matrices:

$$
Y_{n}=\left[\begin{array}{cc}
P_{n+1} & P_{n}^{(1)} \\
P_{n} & P_{n-1}^{(1)}
\end{array}\right], \tilde{Y}_{n}=\left[\begin{array}{cc}
P_{n+1} & q_{n+1} / w \\
P_{n} & q_{n} / w
\end{array}\right], Q_{n}=\left[\begin{array}{c}
q_{n+1} \\
q_{n}
\end{array}\right], n \geq 0 .
$$

Lemma 1. Let $\left\{P_{n}\right\}$ be a SMOP and let $\beta_{n}, \gamma_{n}$ be the coefficients of the three term recurrence relation ( 7$)$. Let $\left\{Y_{n}\right\},\left\{\widetilde{Y}_{n}\right\},\left\{Q_{n}\right\}$ be the sequences defined in (10). Then,

(a) $Y_{n}$ and $\widetilde{Y}_{n}$ satisfy the difference equation

$$
X_{n}=\mathcal{A}_{n} X_{n-1}, \quad \mathcal{A}_{n}=\left[\begin{array}{cc}
x-\beta_{n} & -\gamma_{n} \\
1 & 0
\end{array}\right], \quad n \geq 1
$$

with initial conditions $Y_{0}=\left[\begin{array}{cc}x-\beta_{0} & 1 \\ 1 & 0\end{array}\right], \quad \widetilde{Y}_{0}=\left[\begin{array}{cc}x-\beta_{0} & q_{1} / w \\ 1 & q_{0} / w\end{array}\right]$;

(b) $Q_{n}$ satisfies

$$
Q_{n}=\mathcal{A}_{n} Q_{n-1}, \quad n \geq 1
$$

with $\mathcal{A}_{n}$ given in (11) and initial conditions $Q_{0}=\left[\begin{array}{c}\left(x-\beta_{0}\right) S-1 \\ S\end{array}\right]$.

Throughout the paper $I$ denotes the $2 \times 2$ identity matrix. The $(i, j)$ entry of a matrix $X$ will be denoted by $X^{(i, j)}$. 


\section{Characterization in terms of matrix Sylvester differ- ential equations}

Theorem 1. Let $S$ be a Stieltjes function, let $\left\{Y_{n}\right\}$ and $\left\{Q_{n}\right\}$ be the corresponding sequences defined in (10), and let $\beta_{n}, \gamma_{n}$ be the corresponding recurrence relation coefficients. The following statements are equivalent:

(a) $S$ satisfies

$$
A S^{\prime}=B S^{2}+C S+D, A, B, C, D \in \mathbb{P}
$$

(b) $Y_{n}$ satisfies the matrix Sylvester equation

$$
A Y_{n}^{\prime}=\mathcal{B}_{n} Y_{n}-Y_{n} \mathcal{C}, \quad n \geq 0,
$$

where

$$
\mathcal{B}_{n}=\left[\begin{array}{cc}
l_{n} & \Theta_{n} \\
-\Theta_{n-1} / \gamma_{n} & l_{n-1}+\left(x-\beta_{n}\right) \Theta_{n-1} / \gamma_{n}
\end{array}\right], \quad \mathcal{C}=\left[\begin{array}{cc}
C / 2 & -D \\
B & -C / 2
\end{array}\right]
$$

with $l_{n}, \Theta_{n}$ polynomials of uniformly bounded degrees, satisfying the initial conditions

$$
\begin{array}{r}
A=\left(l_{0}-C / 2\right)\left(x-\beta_{0}\right)-B+\Theta_{0}, 0=D\left(x-\beta_{0}\right)+l_{0}+C / 2, \\
\Theta_{-1}=D, l_{-1}=C / 2 ;
\end{array}
$$

(c) the matrices defined in (11), $\mathcal{A}_{n}=\left[\begin{array}{cc}x-\beta_{n} & -\gamma_{n} \\ 1 & 0\end{array}\right]$, satisfy

$$
A \mathcal{A}_{n}^{\prime}=\mathcal{B}_{n} \mathcal{A}_{n}-\mathcal{A}_{n} \mathcal{B}_{n-1}, \quad n \geq 1
$$

(d) $Q_{n}$ satisfies

$$
A Q_{n}^{\prime}=\left(\mathcal{B}_{n}+(B S+C / 2) I\right) Q_{n}, \quad n \geq 0 .
$$

Proof: Taking into account [3, Theorem 2] we only need to prove $(b) \Leftrightarrow(c)$. (b) $\Rightarrow(c)$. To obtain (15) we take derivatives on $Y_{n}=\mathcal{A}_{n} Y_{n-1}$ (cf. (11)), and substitute it in (13), thus obtaining

$$
A \mathcal{A}_{n}^{\prime} Y_{n-1}+A \mathcal{A}_{n} Y_{n-1}^{\prime}=\mathcal{B}_{n} Y_{n}-Y_{n} \mathcal{C} .
$$

Using (13) for $n-1$ in the previous equation we get

$$
A \mathcal{A}_{n}^{\prime} Y_{n-1}+\mathcal{A}_{n}\left(\mathcal{B}_{n-1} Y_{n-1}-Y_{n-1} \mathcal{C}\right)=\mathcal{B}_{n} Y_{n}-Y_{n} \mathcal{C} .
$$

Using the recurrence relation (11) for $Y_{n}$ we obtain

$$
A \mathcal{A}_{n}^{\prime} Y_{n-1}+\mathcal{A}_{n}\left(\mathcal{B}_{n-1} Y_{n-1}-Y_{n-1} \mathcal{C}\right)=\mathcal{B}_{n} \mathcal{A}_{n} Y_{n-1}-\mathcal{A}_{n} Y_{n-1} \mathcal{C},
$$


that is,

$$
A \mathcal{A}_{n}^{\prime} Y_{n-1}=\left(\mathcal{B}_{n} \mathcal{A}_{n}-\mathcal{A}_{n} \mathcal{B}_{n-1}\right) Y_{n-1} .
$$

Since $Y_{n}$ is nonsingular, for all $n \geq 0$, there follows (15).

$(c) \Rightarrow(b)$. If we multiply (15) by $Y_{n-1}$, we obtain

$$
A\left(\mathcal{A}_{n} Y_{n-1}\right)^{\prime}-A \mathcal{A}_{n} Y_{n-1}^{\prime}=\mathcal{B}_{n} \mathcal{A}_{n} Y_{n-1}-\mathcal{A}_{n} \mathcal{B}_{n-1} Y_{n-1}
$$

Taking into account the recurrence relation (11) for $Y_{n}$ we get

$$
A Y_{n}^{\prime}-\mathcal{B}_{n} Y_{n}=\mathcal{A}_{n}\left(A Y_{n-1}^{\prime}-\mathcal{B}_{n-1} Y_{n-1}\right),
$$

thus

$$
A Y_{n}^{\prime}-\mathcal{B}_{n} Y_{n}=\mathcal{A}_{n} \cdots \mathcal{A}_{2}\left(A Y_{1}^{\prime}-\mathcal{B}_{1} Y_{1}\right) .
$$

The use of $\mathcal{A}_{n} \cdots \mathcal{A}_{2}=Y_{n} Y_{1}^{-1}$ in the preceding equation yields an equation for $Y_{n}$ of the Sylvester type (13), $A Y_{n}^{\prime}=\mathcal{B}_{n} Y_{n}-Y_{n} \widetilde{C}$, with $\widetilde{C}=-Y_{1}^{-1}\left(A Y_{1}^{\prime}-\right.$ $\left.\mathcal{B}_{1} Y_{1}\right)$.

Corollary 1. The following relations hold:

$$
\begin{gathered}
\operatorname{tr}\left(\mathcal{B}_{n}\right)=0, \quad n \geq 0, \\
\operatorname{det}\left(\mathcal{B}_{n}\right)=\operatorname{det}\left(\mathcal{B}_{0}\right)+A \sum_{k=1}^{n} \frac{\Theta_{k-1}}{\gamma_{k}}, n \geq 1,
\end{gathered}
$$

and $\operatorname{det}\left(\mathcal{B}_{0}\right)=D(A+B)-(C / 2)^{2}$.

Proof: The formulas for the trace and determinant of the matrices $\mathcal{B}_{n}$ involved in equations of the same type as (15) were deduced in [2, Lemma 2.5]. The use of the initial conditions (14) yields (16) and (17).

Remark 1. Since $\operatorname{tr}\left(\mathcal{B}_{n}\right)=0$, henceforth we parameterize the matrix $\mathcal{B}_{n}$ in terms of the two functions $l_{n}$ and $\Theta_{n}$ as $\mathcal{B}_{n}=\left[\begin{array}{cc}l_{n} & \Theta_{n} \\ -\Theta_{n-1} / \gamma_{n} & -l_{n}\end{array}\right]$.

Remark 2. Equation (15) reads as

$$
\left\{\begin{array}{l}
\left(x-\beta_{n}\right)\left(l_{n}-l_{n-1}\right)=A-\Theta_{n}+\frac{\gamma_{n}}{\gamma_{n-1}} \Theta_{n-2} \\
l_{n}-l_{n-2}=-\frac{\left(x-\beta_{n}\right)}{\gamma_{n}} \Theta_{n-1}+\frac{\left(x-\beta_{n-1}\right)}{\gamma_{n-1}} \Theta_{n-2}, \quad n \geq 1,
\end{array}\right.
$$

which are comparable with the so-called Laguerre-Freud's equations [12, 13]. 
3.1. Characterization of semi-classical orthogonal polynomials. As a consequence of the previous theorem we deduce the characterization that follows.

Theorem 2. Let $\left\{P_{n}\right\}$ be a SMOP with respect to a weight $w$, and let $\left\{q_{n}\right\}$ be the corresponding sequence of functions of the second kind. The weight $w$ is semi-classical and satisfies $w^{\prime} / w=C / A$ if, and only if, $\widetilde{Y}_{n}=$ $\left[\begin{array}{cc}P_{n+1} & q_{n+1} / w \\ P_{n} & q_{n} / w\end{array}\right]$ satisfies the matrix differential equation

$$
A \widetilde{Y}_{n}^{\prime}=\left(\mathcal{B}_{n}-\frac{C}{2} I\right) \widetilde{Y}_{n}, \quad n \geq 1
$$

where $\mathcal{B}_{n}$ is the matrix associated with the equation $A S^{\prime}=C S+D$ for the Stieltjes function of $w$.

Proof: Note that $w^{\prime} / w=C / A$ implies $A S^{\prime}=C S+D, D \in \mathbb{P}$, for the corresponding Stieltjes function (see [15]). Taking into account the Theorem 1 we get

$$
\left\{\begin{array}{l}
A P_{n+1}^{\prime}=\left(l_{n}-C / 2\right) P_{n+1}+\Theta_{n} P_{n} \\
A q_{n+1}^{\prime}=\left(l_{n}+C / 2\right) q_{n+1}+\Theta_{n} q_{n}, \quad n \geq 0 .
\end{array}\right.
$$

Therefore, using the three term recurrence relation (7) for $\left\{P_{n}\right\}$ we obtain

$$
A P_{n}^{\prime}=\left(l_{n-1}-C / 2+\frac{\left(x-\beta_{n}\right)}{\gamma_{n}} \Theta_{n-1}\right) P_{n}-\frac{\Theta_{n-1}}{\gamma_{n}} P_{n+1}, \quad n \geq 1 .
$$

From (19) there follows

$$
A\left(\frac{q_{n+1}}{w}\right)^{\prime}=\Theta_{n} \frac{q_{n}}{w}+\left(l_{n}-C / 2\right) \frac{q_{n+1}}{w},
$$

where we used $w^{\prime} / w=C / A$. Furthermore, using the three term recurrence relation for $\left\{q_{n}\right\}$ (cf. (12)) we obtain

$$
A\left(\frac{q_{n}}{w}\right)^{\prime}=\left(l_{n-1}-C / 2+\frac{\left(x-\beta_{n}\right)}{\gamma_{n}} \Theta_{n-1}\right) \frac{q_{n}}{w}-\frac{\Theta_{n-1}}{\gamma_{n}} \frac{q_{n+1}}{w}, \quad n \geq 1 .
$$

Equations. (19)-(22) yield

$$
A \widetilde{Y}_{n}^{\prime}=\widetilde{\mathcal{B}}_{n} \widetilde{Y}_{n}, \widetilde{\mathcal{B}}_{n}=\left[\begin{array}{cc}
l_{n}-C / 2 & \Theta_{n} \\
-\Theta_{n-1} / \gamma_{n} & l_{n-1}+\left(x-\beta_{n}\right) \Theta_{n-1} / \gamma_{n}-C / 2
\end{array}\right], n \geq 1,
$$

thus we get (18). 
Conversely, if $\widetilde{Y}_{n}$ satisfies (18), then

$$
\left(\operatorname{det}\left(\widetilde{Y}_{n}\right)\right)^{\prime}=\frac{\operatorname{tr}\left(\mathcal{B}_{n}-C / 2 I\right)}{A} \operatorname{det}\left(\widetilde{Y}_{n}\right) .
$$

Since $\operatorname{det}\left(\widetilde{Y}_{n}\right)=\left(\gamma_{1} \ldots \gamma_{n}\right) / w$ and $\operatorname{tr}\left(\mathcal{B}_{n}-C / 2 I\right)=-C$, there follows $w^{\prime} / w=$ $C / A$, thus $w$ is semi-classical.

Remark 3. The analogue result for orthonormal polynomials was established in [13].

\section{Matrix Sylvester equations and Radon's Lemma}

The theorem that follows is a particular case of the result known, in the literature of matrix Riccati equations, as Radon's Lemma [10, 16].

Theorem 3. Let $A$ be a polynomial, let $\mathcal{B}_{n} / A, n \geq 1$, and $\mathcal{C} / A$ be matrices whose entries are integrable functions in a domain $G$ of the complex plane, and let $x_{0} \in G$. If the matrices $\mathcal{P}_{n}$ and $\mathcal{L}, \mathcal{L}$ nonsingular, satisfy

$$
\left\{\begin{array}{l}
A \mathcal{L}^{\prime}=\mathcal{C} \mathcal{L} \\
\mathcal{L}\left(x_{0}\right)=I
\end{array}\right.
$$

and

$$
\left\{\begin{array}{l}
A \mathcal{P}_{n}^{\prime}=\mathcal{B}_{n} \mathcal{P}_{n}, \quad n \geq 1 \\
\mathcal{P}_{n}\left(x_{0}\right)=Y_{n}\left(x_{0}\right)
\end{array}\right.
$$

then the solution of $A Y_{n}^{\prime}=\mathcal{B}_{n} Y_{n}-Y_{n} \mathcal{C}$, in $G$, is given by:

$$
Y_{n}=\mathcal{P}_{n} \mathcal{L}^{-1}, \quad n \geq 1 .
$$

Our aim is to find a representation for $Y_{n}$ satisfying (13), $A Y_{n}^{\prime}=\mathcal{B}_{n} Y_{n}-Y_{n} \mathcal{C}$, related to $A S^{\prime}=B S^{2}+C S+D$ (cf. Theorem 1). To that end we start with some remarks on the solution of the corresponding problem (24). Note that we are searching for matrices $\mathcal{P}_{n}$ of order two satisfying

$$
A \mathcal{P}_{n}^{\prime}=\mathcal{B}_{n} \mathcal{P}_{n}
$$

Hereafter we will consider $x_{1} \in \mathbb{C}$ and $\tilde{C}$ a polynomial such that $\int_{x_{1}}^{x} \frac{\tilde{C}(t)}{2 A(t)} d t$ is defined in suitable domains.

Lemma 2. Let $\mathcal{B}_{n}$ be the matrices given in (13), and let $\tilde{C}$ be a polynomial. A matrix $\widetilde{Y}_{n}$ satisfies

$$
A \widetilde{Y}_{n}^{\prime}=\left(\mathcal{B}_{n}-\tilde{C} / 2 I\right) \widetilde{Y}_{n}
$$

if, and only if, $\mathcal{P}_{n}=e^{\int_{x_{1}}^{x} \frac{\tilde{C}}{2 A} d t} \widetilde{Y}_{n}$ satisfies (25). 
Taking into account the previous Lemma, we will solve (25) by considering

$$
\mathcal{P}_{n}=e^{\int_{x_{1}}^{x} \frac{\tilde{C}}{2 A} d t} \widetilde{Y}_{n},
$$

where $\widetilde{Y}_{n}$ satisfies (26). Furthermore, taking into account the Theorem 2, we will search for $\widetilde{Y}_{n}$ defined by

$$
\widetilde{Y}_{n}=\left[\begin{array}{cc}
\tilde{P}_{n+1} & \tilde{q}_{n+1} / \tilde{w} \\
\tilde{P}_{n} & \tilde{q}_{n} / \tilde{w}
\end{array}\right]
$$

with $\left\{\tilde{P}_{n}\right\}$ a SMOP with respect to a weight function $\tilde{w}$ and $\left\{\tilde{q}_{n}\right\}$ the corresponding sequence of functions of the second kind.

Remark 4. Note that (26) implies $\operatorname{det}\left(\widetilde{Y}_{n}\right)^{\prime}=\frac{\operatorname{tr}\left(\mathcal{B}_{n}-\tilde{C} / 2 I\right)}{A} \widetilde{Y}_{n}$, which combined with $(27)$ yields $\tilde{w}^{\prime} / \tilde{w}=\tilde{C} / A$, thus $\tilde{w}=e^{\int \tilde{C} / A}$.

Lemma 3. Let $S$ be a Stieltjes function that satisfies the Riccati differential equation $A S^{\prime}=B S^{2}+C S+D$. Let $\left\{P_{n}\right\}$ be the corresponding SMOP such that the equations (13), $A Y_{n}^{\prime}=\mathcal{B}_{n} Y_{n}-Y_{n} \mathcal{C}$, hold, and let (24) be the corresponding system $A \mathcal{P}_{n}^{\prime}=\mathcal{B}_{n} \mathcal{P}_{n}$. Let the following assumption hold:

$$
\exists \tilde{C} \in \mathbb{P}, \exists n_{0} \geq 1: \mathcal{P}_{n}=e^{\int_{x_{1}}^{x} \frac{\tilde{C}(t)}{2 A(t)} d t} \widetilde{Y}_{n-n_{0}}, \quad n \geq n_{0}+1,
$$

with the $\widetilde{Y}_{n}$ 's given as in (27), related to a SMOP $\left\{\tilde{P}_{n}\right\}$. Denote by $\tilde{S}$ the Stieltjes functions related to $\left\{\tilde{P}_{n}\right\}$. Then, the following statements hold:

(a) $\tilde{S}$ is a fractional linear transformation of $S$,

$$
\tilde{S}=\frac{a+b S}{c+d S}, \quad a, b, c, d \in \mathbb{P}
$$

(b) $\tilde{S}$ satisfies

$$
A \tilde{S}^{\prime}=\tilde{C} \tilde{S}+\tilde{D}, \quad \tilde{D} \in \mathbb{P},
$$

the polynomials $\tilde{C}, \tilde{D}$ being related to $B, C, D$ by

$$
\begin{aligned}
(b c-a d) B & =\left(b d^{\prime}-b^{\prime} d\right) A+b d \tilde{C}+d^{2} \tilde{D} \\
(b c-a d) C & =\left(b c^{\prime}+a d^{\prime}-b^{\prime} c-a^{\prime} d\right) A+(a d+b c) \tilde{C}+2 c d \tilde{D}, \\
(b c-a d) D & =\left(a c^{\prime}-a^{\prime} c\right) A+a c \tilde{C}+c^{2} \tilde{D} .
\end{aligned}
$$

Proof: (a). Let us denote by $\tilde{\mathcal{A}}_{n}$ the matrices of the recurrence relation of $\left\{\widetilde{Y}_{n}\right\}$. From $\mathcal{P}_{n}=e^{\int_{z_{1}}^{z} \frac{\tilde{C}}{2 A} d t} \widetilde{Y}_{n-n_{0}}$ and $\widetilde{Y}_{n-n_{0}}=\tilde{\mathcal{A}}_{n-n_{0}} \widetilde{Y}_{n-n_{0}-1}$ there follows

$$
\mathcal{P}_{n}=\tilde{\mathcal{A}}_{n-n_{0}} \mathcal{P}_{n-1}, \quad n \geq n_{0}+1 \text {. }
$$


The substitution of (34) into $A \mathcal{P}_{n}^{\prime}=\mathcal{B}_{n} \mathcal{P}_{n}$ yields

$$
A\left(\tilde{\mathcal{A}}_{n-n_{0}} \mathcal{P}_{n-1}\right)^{\prime}=\mathcal{B}_{n} \tilde{\mathcal{A}}_{n-n_{0}} \mathcal{P}_{n-1}, \quad n \geq n_{0}+1,
$$

from which we obtain

$$
A \tilde{\mathcal{A}}_{n-n_{0}}^{\prime}=\mathcal{B}_{n} \tilde{\mathcal{A}}_{n-n_{0}}-\tilde{\mathcal{A}}_{n-n_{0}} \mathcal{B}_{n-1}, \quad n \geq n_{0}+1 .
$$

Using the equations enclosed in the positions $(2,1)$ and $(2,2)$ of $(35)$ we obtain

$$
\tilde{\gamma}_{n-n_{0}}=\gamma_{n}, \quad \tilde{\beta}_{n-n_{0}}=\beta_{n}, \quad n \geq n_{0}+1 .
$$

Consequently, taking into account the representation of the Stieltjes function in terms of a continued fraction (8), (29) follows.

(b). From (28) we obtain that $\left\{\tilde{P}_{n}\right\}$ is semi-classical, and the corresponding $\tilde{w}$ satisfies $\tilde{w}^{\prime} / \tilde{w}=\tilde{C} / A$ (note the remark 4 ). Thus, the first order differential equation (30) for the corresponding Stieltjes function, $\tilde{S}$, follows.

By substituting $\tilde{S}$ given by (29) in (30) we get

$$
A(b c-a d) S^{\prime}=B_{1} S^{2}+C_{1} S+D_{1}
$$

with

$$
\begin{aligned}
& B_{1}=\left(b d^{\prime}-b^{\prime} d\right) A+b d \tilde{C}+d^{2} \tilde{D}, \\
& C_{1}=\left(b c^{\prime}+a d^{\prime}-b^{\prime} c-a^{\prime} d\right) A+(a d+b c) \tilde{C}+2 c d \tilde{D}, \\
& D_{1}=\left(a c^{\prime}-a^{\prime} c\right) A+a c \tilde{C}+c^{2} \tilde{D} .
\end{aligned}
$$

Since $S$ satisfies $A S^{\prime}=B S^{2}+C S+D$ as well as (36), there follows, if $A-D$ and $B_{1}-D_{1}$ are non vanishing, that

$$
\varrho=\frac{B}{B_{1}}=\frac{C}{C_{1}}=\frac{D}{D_{1}}, \varrho=1 /(b c-a d) .
$$

Hence, we obtain (31)-(33).

Theorem 4. Let $S$ be a Stieltjes function satisfying $A S^{\prime}=B S^{2}+C S+D$. To $A S^{\prime}=B S^{2}+C S+D$ associate $A Y_{n}^{\prime}=\mathcal{B}_{n} Y_{n}-Y_{n} \mathcal{C}$ and the corresponding systems (23) and (24), $A \mathcal{L}^{\prime}=\mathcal{C} \mathcal{L}, A \mathcal{P}_{n}^{\prime}=\mathcal{B}_{n} \mathcal{P}_{n}$. Let $G$ be a domain in the complex plane such that the entries of the matrices $\mathcal{B}_{n} / A$ and $\mathcal{C} / A$ are integrable in $G$. Assume that the assumption (28) holds. Then, there exists a polynomial $\tilde{C}$ (defined by (31)-(33)) and a weight function $\tilde{w}=e^{\int \frac{\tilde{C}}{A}}$ such the following representation holds in $G$ :

$$
Y_{n}=\left[\begin{array}{cc}
\sqrt{\tilde{w}} \tilde{P}_{n-n_{0}+1} & \tilde{q}_{n-n_{0}+1} / \sqrt{\tilde{w}} \\
\sqrt{\tilde{w}} \tilde{P}_{n-n_{0}} & \tilde{q}_{n-n_{0}} / \sqrt{\tilde{w}}
\end{array}\right] E_{n} \mathcal{L}^{-1}, \quad n \geq n_{0}+1
$$


with $\left\{\tilde{P}_{n}\right\}$ the SMOP with respect to $\tilde{w},\left\{\tilde{q}_{n}\right\}$ the corresponding sequence of functions of the second kind, and $E_{n}=\mathcal{P}_{n}\left(x_{0}\right)^{-1} Y_{n}\left(x_{0}\right), x_{0} \in G$.

Proof: The result follows from the Theorem 3 combined with the Lemma 3 (note the remark 4).

\section{Laguerre-Hahn orthogonal polynomials of class zero}

Laguerre-Hahn orthogonal polynomials of class zero are related to Stieltjes functions, $S$, satisfying

$$
A S^{\prime}=B S^{2}+C S+D, \operatorname{deg}(C)=1, \quad \max \{\operatorname{deg}(A), \operatorname{deg}(B)\} \leq 2 .
$$

There are, up to a linear change of variable, three canonical cases for the triples of polynomials satisfying the above Riccati differential equation, according to the degree of $A$ be zero, one or two [1].

In subsection 5.1 we will show the solutions of the corresponding difference equation (15) in the class zero: we give closed form expressions for the recurrence relation coefficients of the orthogonal polynomials. In subsection 5.2 we will use Radon's Lemma in order to obtain representations for a sequence of Laguerre-Hahn orthogonal polynomials.

\subsection{The recurrence relation coefficients.}

Theorem 5. Let $S$ be a Stieltjes function satisfying $A S^{\prime}=B S^{2}+C S+D$ with $\operatorname{deg}(C)=1, \max \{\operatorname{deg}(A), \operatorname{deg}(B)\} \leq 2$. Let $A(x)=a_{2} x^{2}+a_{1} x+a_{0}, B(x)=$ $b_{2} x^{2}+b_{1} x+b_{0}, C(x)=c_{1} x+c_{0}$. The recurrence relation coefficients of the $S M O P\left\{P_{n}\right\}$ related to $S$ are given by

$$
\begin{aligned}
\beta_{n} & =\frac{\left(r_{1}^{2}-a_{2}^{2}\right) \beta_{1}+2 a_{1}(n-1)\left(r_{1}-(n-1) a_{2}\right)}{\left(r_{1}-(2 n-1) a_{2}\right)\left(r_{1}-(2 n-3) a_{2}\right)}, \quad n \geq 2, \\
\gamma_{n+1} & =\frac{r_{1}\left(r_{1}-2 a_{2}\right) \gamma_{2}+\sum_{k=2}^{n} A\left(\beta_{k}\right)\left(r_{1}-2(k-1) a_{2}\right)}{\left(r_{1}-2 n a_{2}\right)\left(r_{1}-2(n-1) a_{2}\right)}, \quad n \geq 2,
\end{aligned}
$$

where

$$
\begin{gathered}
\beta_{1}=\frac{a_{1}+2 \beta_{0} D-c_{0}}{r_{1}-a_{2}}, \quad r_{1}=\Theta_{0} / \gamma_{1}, \quad \Theta_{0}=A+B+\left(x-\beta_{0}\right)^{2} D+\left(x-\beta_{0}\right) C, \\
\gamma_{1}=\frac{\Theta_{0}}{2 D+c_{1}-a_{2}}, \quad \gamma_{2}=\frac{A\left(\beta_{1}\right)+\gamma_{1} D}{r_{1}-2 a_{2}} .
\end{gathered}
$$


Proof: The corresponding equations (15) read as

$$
\left\{\begin{array}{l}
\left(x-\beta_{n}\right)\left(l_{n}-l_{n-1}\right)=A-\Theta_{n}+\frac{\gamma_{n}}{\gamma_{n-1}} \Theta_{n-2} \\
l_{n}-l_{n-2}=-\frac{\left(x-\beta_{n}\right)}{\gamma_{n}} \Theta_{n-1}+\frac{\left(x-\beta_{n-1}\right)}{\gamma_{n-1}} \Theta_{n-2}, \quad n \geq 1,
\end{array}\right.
$$

where the initial conditions hold (cf. (14)):

$$
\begin{aligned}
& l_{-1}(x)=C(x) / 2, l_{0}(x)=-\left(x-\beta_{0}\right) D-C(x) / 2, \\
& \Theta_{-1}=D, \Theta_{0}=A(x)+B(x)+\left(x-\beta_{0}\right)^{2} D+\left(x-\beta_{0}\right) C(x) .
\end{aligned}
$$

Note that under the stated conditions on the degrees of $A, B, C$, one has

$$
l_{n}(x)=\ell_{n, 1} x+\ell_{n, 0}, \quad \Theta_{n}, D \text { constants. }
$$

By substituting (41) into (40) and equating the coefficients of $x^{2}, x, x^{0}$ we obtain, for all $n \geq 1$,

$$
\begin{aligned}
\ell_{n, 1}-\ell_{n-1,1} & =a_{2} \\
\ell_{n, 0}-\ell_{n-1,0} & =\beta_{n}\left(\ell_{n, 1}-\ell_{n-1,1}\right)+a_{1}, \\
-\beta_{n}\left(\ell_{n, 0}-\ell_{n-1,0}\right) & =a_{0}-\Theta_{n}+\frac{\gamma_{n}}{\gamma_{n-1}} \Theta_{n-2}, \\
\ell_{n, 1}-\ell_{n-2,1} & =-\frac{\Theta_{n-1}}{\gamma_{n}}+\frac{\Theta_{n-2}}{\gamma_{n-1}}, \\
\ell_{n, 0}-\ell_{n-2,0} & =\beta_{n} \frac{\Theta_{n-1}}{\gamma_{n}}-\beta_{n-1} \frac{\Theta_{n-2}}{\gamma_{n-1}} .
\end{aligned}
$$

From (42) and (43) there follows

$$
\begin{aligned}
& \ell_{n, 1}=\ell_{0,1}+n a_{2}, \quad n \geq 1 \\
& \ell_{n, 0}=\ell_{0,0}+a_{2} \sum_{k=1}^{n} \beta_{k}+n a_{1}, \quad n \geq 1 .
\end{aligned}
$$

Note that (46) and (47) are also valid for $n=0$, using the convention $\sum_{k=i}^{j} \cdot=$ 0 whenever $i>j$.

The use of (46) for $n$ and $n-2$ in (44) yields

$$
\frac{\Theta_{n-1}}{\gamma_{n}}=\frac{\Theta_{n-2}}{\gamma_{n-1}}-2 a_{2}, \quad n \geq 2,
$$

thus

$$
\frac{\Theta_{n-1}}{\gamma_{n}}=\frac{\Theta_{0}}{\gamma_{1}}-2(n-1) a_{2}, n \geq 2
$$


Note that the above equality also holds for $n=1$, thus we get

$$
\Theta_{n-1}=\left(r_{1}-2(n-1) a_{2}\right) \gamma_{n}, \quad r_{1}=\frac{\Theta_{0}}{\gamma_{1}}, \quad n \geq 1 .
$$

The use of (47) for $n$ and $n-2$ as well as (48) for $n$ and $n-1$ in (45) yields $a_{2}\left(\beta_{n-1}+\beta_{n}\right)+2 a_{1}=\beta_{n}\left(r_{1}-2(n-1) a_{2}\right)-\beta_{n-1}\left(r_{1}-2(n-2) a_{2}\right), \quad n \geq 2$, that is,

$$
\beta_{n}\left(r_{1}-(2 n-1) a_{2}\right)=\beta_{n-1}\left(r_{1}-(2 n-5) a_{2}\right)+2 a_{1}, \quad n \geq 2 .
$$

If we multiply the above equation by $r_{1}-(2 n-3) a_{2}$ we get

$$
\begin{gathered}
L_{n+1}=L_{n}+2 a_{1}\left(r_{1}-(2 n-3) a_{2}\right), \\
L_{n}=\beta_{n-1}\left(r_{1}-(2 n-3) a_{2}\right)\left(r_{1}-(2 n-5) a_{2}\right) .
\end{gathered}
$$

Therefore, we obtain

$$
L_{n+1}=L_{2}+2 a_{1} \sum_{k=2}^{n}\left(r_{1}-(2 k-3) a_{2}\right), \quad n \geq 2,
$$

thus for $n \geq 2$

$\beta_{n}\left(r_{1}-(2 n-1) a_{2}\right)\left(r_{1}-(2 n-3) a_{2}\right)=\beta_{1}\left(r_{1}^{2}-a_{2}^{2}\right)+2 a_{1}(n-1)\left(r_{1}-(n-1) a_{2}\right)$, thus (37) follows.

To obtain an equation for the $\gamma$ 's we start by evaluating the first equation of (40) at $\beta_{n}$, thus obtaining

$$
\Theta_{n}-\frac{\gamma_{n}}{\gamma_{n-1}} \Theta_{n-2}=A\left(\beta_{n}\right), \quad n \geq 1 .
$$

Using (48) for $n+1$ and $n-1$ in the above equation we get

$$
\gamma_{n+1}\left(r_{1}-2 n a_{2}\right)-\gamma_{n}\left(r_{1}-2(n-2) a_{2}\right)=A\left(\beta_{n}\right), \quad n \geq 2 .
$$

If we multiply the above equation by $r_{1}-2(n-1) a_{2}$ we get for $n \geq 2$ $T_{n+1}=T_{n}+A\left(\beta_{n}\right)\left(r_{1}-2(n-1) a_{2}\right), T_{n}=\gamma_{n}\left(r_{1}-2(n-2) a_{2}\right)\left(r_{1}-2(n-1) a_{2}\right)$.

Therefore, we obtain

$$
T_{n+1}=T_{2}+\sum_{k=2}^{n} A\left(\beta_{k}\right)\left(r_{1}-2(k-1) a_{2}\right), \quad n \geq 2,
$$

thus (38) follows.

(39) follows from the initial conditions. 


\subsection{Representations of Laguerre-Hahn orthogonal polynomials of class zero via Radon's Lemma: an example.}

Lemma 4. Let $\left\{P_{n}\right\}$ be a $S M O P$ related to $A S^{\prime}=B S^{2}+C S+D, A(x)=$ $a_{2} x^{2}+a_{1} x+a_{0}, B(x)=b_{2} x^{2}+b_{1} x+b_{0}, C(x)=c_{1} x+c_{0}$. Then, the matrices $\mathcal{B}_{n}=\left[\begin{array}{cc}l_{n} & \Theta_{n} \\ -\Theta_{n-1} / \gamma_{n} & -l_{n}\end{array}\right]$ involved in the corresponding equations (13) are defined by $l_{n}(x)=\ell_{n, 1} x+\ell_{n, 0}$ and $\Theta_{n}$ constant, where, for all $n \geq 1$,

$$
\begin{aligned}
\ell_{n, 1}= & c_{1} / 2+(n+1) a_{2}+b_{2}, \\
\ell_{n, 0}= & c_{0} / 2+(n+1) a_{1}+b_{1}+\beta_{0}\left(b_{2}+a_{2}\right)+\alpha_{n} a_{2}, \\
\Theta_{n}= & \left(\ell_{n, 0}+c_{0} / 2-(n-1) a_{1}\right) \alpha_{n}-\left(\ell_{n, 1}+c_{1} / 2-(n-2) a_{2}\right) \nu_{n} \\
& \quad-\left(\nu_{n}+\beta_{0} \alpha_{n}-\gamma_{1}\right) D+n a_{0},
\end{aligned}
$$

and

$$
\begin{aligned}
\ell_{0,1}=-D-c_{1} / 2, & \ell_{0,0}=\beta_{0} D-c_{0} / 2, \\
\Theta_{0} & =A+B+\left(x-\beta_{0}\right)^{2} D+\left(x-\beta_{0}\right) C, \quad \Theta_{-1}=D,
\end{aligned}
$$

with

$$
D=-a_{2}-c_{1}-b_{2}, \quad \alpha_{n}=\sum_{k=1}^{n} \beta_{k}, \quad \nu_{n}=\sum_{1 \leq i<j \leq n}^{n} \beta_{i} \beta_{j}-\sum_{k=2}^{n} \gamma_{k}, \quad n \geq 1 .
$$

Proof: Write

$$
\begin{aligned}
P_{n}^{(1)}(x) & =x^{n}-\alpha_{n} x^{n-1}+\nu_{n} x^{n-2}+\cdots \\
P_{n+1}(x) & =x^{n+1}-\left(\alpha_{n}+\beta_{0}\right) x^{n}+\left(\nu_{n}+\beta_{0} \alpha_{n}-\gamma_{1}\right) x^{n-1}+\cdots
\end{aligned}
$$

and compare the coefficients of the corresponding monomials. To obtain $l_{0}(x), \Theta_{0}$, and $\Theta_{-1}$, use the initial conditions (14).

For later purposes we show some results concerning the Hermite polynomials.

Lemma 5. Let $\left\{H_{n}\right\}$ denote the sequence of monic Hermite polynomials, and let $\left\{\tilde{q_{n}}\right\}$ be the corresponding sequence of functions of the second kind. The following statements hold:

(a) $\left\{H_{n}\right\}$ is related to the Stieltjes function $\tilde{S}$ satisfying

$$
A \tilde{S}^{\prime}=\tilde{C} \tilde{S}+\tilde{D}, \quad A=1, \tilde{C}=-2 x, \tilde{D}=2 ;
$$


(b) the recurrence relation coefficients of $\left\{H_{n}\right\}$ are given by

$$
\tilde{\beta}_{n}=0, \tilde{\gamma}_{n+1}=(n+1) / 2, \quad n \geq 0 ;
$$

(c) $\widetilde{H}_{n}=\left[\begin{array}{cc}H_{n+1} & \tilde{q}_{n+1} / \tilde{w} \\ H_{n} & \tilde{q}_{n} / \tilde{w}\end{array}\right]$, with $\tilde{w}=e^{-x^{2}}$, satisfies the differential system

$$
\widetilde{H}_{n}^{\prime}=\left(\tilde{\mathcal{B}}_{n}-\frac{\tilde{C}}{2} I\right) \widetilde{H}_{n}, \quad \tilde{\mathcal{B}}_{n}=\left[\begin{array}{cc}
-x & n+1 \\
-2 & x
\end{array}\right], \quad n \geq 1 .
$$

Proof: (53) is given in [15]. (54) follows from (37) and (38). To obtain (55) we use the Theorem 2 (cf. (18)), where the entries of $\tilde{\mathcal{B}}_{n}$ can be obtained using (49)-(51).

Lemma 6. Let $\left\{P_{n}\right\}$ be the SMOP related to the Stieltjes function $S$ satisfying $A S^{\prime}=B S^{2}+C S+D$, where

$$
A=1, B=-2 x^{2}+2 \lambda x+\rho-1, C=2 x, D=0,
$$

with $\rho=2 \gamma_{1}, \lambda=\beta_{0}$, and $\rho \lambda \neq 0$. Let $\left\{Y_{n}=\left[\begin{array}{cc}P_{n+1} & P_{n}^{(1)} \\ P_{n} & P_{n-1}^{(1)}\end{array}\right]\right\}$ be the sequence associated with $\left\{P_{n}\right\}$ defined in (10). The following statements hold:

(a) the recurrence relation coefficients of $\left\{P_{n}\right\}$ are given by

$$
\beta_{n}=0, \gamma_{n+1}=n / 2, \quad n \geq 1
$$

(b) $Y_{n}$ satisfies the matrix Sylvester equations $Y_{n}^{\prime}=\mathcal{B}_{n} Y_{n}-\mathcal{C} Y_{n}, n \geq 0$, with

$$
\mathcal{B}_{0}=\left[\begin{array}{cc}
-x & \rho \\
0 & x
\end{array}\right], \quad \mathcal{B}_{n}=\left[\begin{array}{ll}
-x & n \\
-2 & x
\end{array}\right], n \geq 1, \quad \mathcal{C}=\left[\begin{array}{cc}
x & 0 \\
B & -x
\end{array}\right]
$$

(c) let $x_{0}$ be an arbitrary point in the complex plane. The solution of the initial value problem

$$
\left\{\begin{array}{l}
\mathcal{L}^{\prime}(x)=\mathcal{C}(x) \mathcal{L}(x) \\
\mathcal{L}\left(x_{0}\right)=I
\end{array}\right.
$$


with $\mathcal{C}$ given in (57), is

$$
\begin{aligned}
& \mathcal{L}^{(1,1)}(x)=e^{-x_{0}^{2} / 2} e^{x^{2} / 2}, \\
& \mathcal{L}^{(1,2)}(x) \equiv 0, \\
& \mathcal{L}^{(2,1)}(x)=e^{-x_{0}^{2} / 2} e^{-x^{2} / 2} \int_{x_{0}}^{x} B(s) e^{s^{2}} d s, \\
& \mathcal{L}^{(2,2)}(x)=e^{x_{0}^{2} / 2} e^{-x^{2} / 2},
\end{aligned}
$$

and the solution of the initial value problem

$$
\left\{\begin{array}{l}
\mathcal{P}_{n}^{\prime}(x)=\mathcal{B}_{n}(x) \mathcal{P}_{n}(x), \quad n \geq 1, \\
\mathcal{P}_{n}\left(x_{0}\right)=Y_{n}\left(x_{0}\right)
\end{array}\right.
$$

with $\mathcal{B}_{n}$ given in (57), is for $n \geq 2$

$$
\mathcal{P}_{n}(x)=e^{-x^{2} / 2} \widetilde{H}_{n-1}(x) \mathcal{K}_{n}, \quad \mathcal{K}_{n}=e^{x_{0}^{2} / 2}\left(\widetilde{H}_{n-1}\left(x_{0}\right)\right)^{-1} Y_{n}\left(x_{0}\right) .
$$

Proof: (56) follows from (37) and (38). To obtain the entries of $\mathcal{B}_{n}$ in (57) use (49)-(52).

Let us solve (63). Since $\widetilde{H}_{n}=\left[\begin{array}{cc}H_{n+1} & \tilde{q}_{n+1} / \tilde{w} \\ H_{n} & \tilde{q}_{n} / \tilde{w}\end{array}\right]$ satisfies (55),

$$
\widetilde{H}_{n}^{\prime}=\left(\tilde{\mathcal{B}}_{n}-\frac{\tilde{C}}{2} I\right) \widetilde{Y}_{n}, \quad \tilde{\mathcal{B}}_{n}=\left[\begin{array}{cc}
-x & n+1 \\
-2 & x
\end{array}\right], \quad \tilde{C}=-2 x, \quad n \geq 1,
$$

then, taking into account the Lemma 2,

$$
\mathcal{P}_{n}=e^{-x^{2} / 2} \widetilde{H}_{n-1}
$$

satisfies $\mathcal{P}_{n}^{\prime}=\tilde{\mathcal{B}}_{n-1} \mathcal{P}_{n}, \quad n \geq 2$, that is, $\mathcal{P}_{n}^{\prime}=\left[\begin{array}{cc}-x & n \\ -2 & x\end{array}\right] \mathcal{P}_{n}, \quad n \geq 2$. Hence, $\mathcal{P}_{n}$ defined by (65) is a fundamental matrix of the differential system in (63). Thus, a solution of the initial value problem (63) is given by (64).

Remark 5. Taking into account (54) and (56), one has $P_{n}^{(1)}=H_{n}, n \geq 1$. Consequently, the matrix $\mathcal{K}_{n}$ given in (64) is such that

$$
\mathcal{K}_{n}^{(1,2)}=e^{x_{0}^{2} / 2}, \mathcal{K}_{n}^{(2,2)}=0 .
$$

Furthermore, using $Y_{n+1}\left(x_{0}\right)=\mathcal{A}_{n+1}\left(x_{0}\right) Y_{n}\left(x_{0}\right)$ (cf. (11)), we obtain

$$
\widetilde{H}_{n-1}\left(x_{0}\right) \mathcal{K}_{n}=\mathcal{A}_{n+1}^{-1}\left(x_{0}\right) \widetilde{H}_{n}\left(x_{0}\right) \mathcal{K}_{n+1}, \quad n \geq 2,
$$


from which we get

$$
\mathcal{K}_{n}^{(1,1)}=\mathcal{K}_{n+1}^{(1,1)}, \quad \mathcal{K}_{n}^{(2,1)}=\mathcal{K}_{n+1}^{(2,1)}, \quad n \geq 2 .
$$

Theorem 6. Let the conditions and the notations of the two previous Lemmas hold. Then,

(a) the following representation holds, for all $n \geq 2$ :

$$
P_{n+1}(x)=e^{-x^{2}}\left(e^{x_{0}^{2} / 2} \mathcal{K}_{n}^{(1,1)}-\int_{x_{0}}^{x} B(s) e^{s^{2}} d s\right) H_{n}(x)+e^{x_{0}^{2} / 2} \mathcal{K}_{n}^{(2,1)} \tilde{q}_{n}(x),
$$

where $x_{0}$ is an arbitrary complex number;

(b) the Stieltjes functions $S$ and $\tilde{S}$ are related through

$$
\tilde{S}=\frac{a+b S}{c+d S}
$$

where

$$
a=-1 / d, b(x)=1 / d x-\lambda / d, c=0, d= \pm \sqrt{\rho / 2} .
$$

Proof: (a). Taking into account the Theorem 3, one has $Y_{n}=\mathcal{P}_{n} \mathcal{L}^{-1}$, where $\mathcal{L}, \mathcal{P}_{n}$, the solutions of the corresponding initial value problems (58) and (63), are given by (59)-(62) and (64), respectively. Therefore, $Y_{n}=\mathcal{P}_{n} \mathcal{L}^{-1}$ yields, in the position $(1,1),(68)$, where we used $(66)$. The position $(2,1)$ gives us (68) for $n-1$, where we used (67). The positions $(1,2)$ and $(2,2)$ of $Y_{n}=\mathcal{P}_{n} \mathcal{L}^{-1}$ yield $P_{n}^{(1)}=H_{n}$ and $P_{n-1}^{(1)}=H_{n-1}$, respectively.

(b). Note that $\tilde{S}$ satisfies (53),

$$
A \tilde{S}^{\prime}=\tilde{C} \tilde{S}+\tilde{D}, \quad A=1, \quad \tilde{C}=-2 x, \quad \tilde{D}=2 .
$$

Taking into account the Lemma 3 , there holds a relation of the type (69), where the polynomials $a-d$ and the polynomials $A, \tilde{C}, \tilde{D}$ are related through the equations (31)-(33). Thus, (70) follows.

\section{References}

[1] H. Bouakkaz and P. Maroni, Description des polynomes orthogonaux de Laguerre-Hahn de classe zero, in "Orthogonal Plynomials and their applications", (C. Brezinski, L. Gori and A. Ronveaux Eds.) J. C. Baltzer A. G. Basel IMACS Annals on Computing and Applied Mathematics, 1991, pp. 189-194.

[2] A. Branquinho and M.N. Rebocho, On the semiclassical character of orthogonal polynomials satisfying structure relations, J. Difference Equ. Appl. 18 (1) (2012) 111-138.

[3] A. Branquinho, A. Foulquié Moreno, A. Paiva, and M.N. Rebocho, Second order differential equations in the Laguerre-Hahn class (submitted).

[4] Y. Chen and M. Ismail, Jacobi polynomials from compatibility conditions, Proc. Amer. Math. Soc. 133 (2004) 465-472. 
[5] T.S. Chihara, An Introduction to Orthogonal Polynomials, Gordon and Breach, New York, 1978.

[6] J. S. Dehesa, F. Marcellán, A. Ronveaux, On orthogonal polynomials with perturbed recurrence relations, J. Comput. Appl. Math. 30 (1990) 203-212.

[7] J. Dini, Sur les formes linéaires et les polynômes orthogonaux de Laguerre-Hahn, Thèse de doctorat, Univ. Pierre et Marie Curie, Paris, 1988 (in French).

[8] W. Hahn, On Differential Equations for Orthogonal Polynomials, Funkcialaj Ek., 21 (1978) $1-9$.

[9] M.E. Ismail, J. Letessier, and G. Valent, Linear birth and death models and associated Laguerre polynomials, J. Approx. Theory, 56 (1988) 337-348.

[10] G. Jank, Matrix Riccati Differential Equations, (A.P.Santana, J.S. Neves e M.P.Oliveira eds.), Textos de Matemática, no. 36, DMUC, 2005.

[11] A.P. Magnus, Riccati acceleration of the Jacobi continued fractions and Laguerre-Hahn polynomials, in "Padé Approximation and its Applications, Proc., Bad Honnef 1883", Lect. Notes in Math. 1071 (H. Werner e H. T. Bunger, eds.), Springer Verlag, Berlin, 1984, pp. 213-230.

[12] A. Magnus, On Freud's equations for exponential weights, J. Approx. Theory 46 (1986) 65-99.

[13] A. Magnus, Painlevé-type differencial equations for the recurrence coefficients of semi-classical orthogonal polynomials, J. Comp. Appl. Math. 57 (1995) 215-237.

[14] F. Marcellán, A. Ronveaux, Co-recursive orthogonal polynomials and fourth order differential equation, J. Comp. Appl. Math. 25 (1) (1989) 105-109.

[15] P. Maroni, Une théorie algébrique des polynômes orthogonaux. Application aux polynômes orthogonaux semi-classiques, in "Orthogonal Plynomials and their applications", (C. Brezinski, L. Gori and A. Ronveaux Eds.) J. C. Baltzer A. G. Basel IMACS Annals on Computing and Applied Mathematics, 1991, pp. 95-130.

[16] J. Radon, Zum Problem von Lagrange, Hamburger Math. Einzelschr., 6, 1928.

[17] A. Ronveaux, Fourth-order differential equations for the numerator polynomials, J. Phys. A.: Math. Gen. 21 (1988), 749-753.

[18] G. Szegő, Orthogonal Polynomials, fourth ed., Amer. Math. Soc. Colloq. Publ., vol. 23, Providence Rhode Island, 1975.

A. Branquinho

CMUC and Department of Mathematics, University of Coimbra, Apartado 3008, EC Santa

Cruz, 3001-501 COIMBRA, Portugal.

E-mail address: ajplb@mat.uc.pt

A. PAIVA

CMUC and Department of Mathematics, University of Beira Interior, 6201-001 Covilhã, Portugal.

E-mail address: apaiva@ubi.pt

M.N. REBOCHO

CMUC and Department of Mathematics, University of Beira Interior, 6201-001 Covilhã, Portugal.

E-mail address: mneves@ubi.pt 